\title{
Effects of Various Extents of High-Frequency Hearing Loss on Speech Recognition and Gap Detection at Low Frequencies in Patients with Sensorineural Hearing Loss
}

\author{
Bei Li, Yang Guo, Guang Yang, Yanmei Feng, and Shankai Yin \\ Department of Otolaryngology Head and Neck Surgery, Shanghai Jiao Tong University Affiliated Sixth People's Hospital, No. 600, \\ Yishan Road, Xuhui District, Shanghai 200233, China
}

Correspondence should be addressed to Yanmei Feng; feng.yanmei@126.com and Shankai Yin; yinshankai@china.com

Received 29 August 2017; Accepted 7 November 2017; Published 27 December 2017

Academic Editor: Hai Huang

Copyright (C) 2017 Bei Li et al. This is an open access article distributed under the Creative Commons Attribution License, which permits unrestricted use, distribution, and reproduction in any medium, provided the original work is properly cited.

\begin{abstract}
This study explored whether the time-compressed speech perception varied with the degree of hearing loss in high-frequency sensorineural hearing loss (HF SNHL) individuals. 65 HF SNHL individuals with different cutoff frequencies were recruited and further divided into mildly, moderately, and/or severely affected subgroups in terms of the averaged thresholds of all frequencies exhibiting hearing loss. Time-compressed speech recognition scores under both quiet and noisy conditions and gap detection thresholds within low frequencies that had normal thresholds were obtained from all patients and compared with data from 11 age-matched individuals with normal hearing threshold at all frequencies. Correlations of the time-compressed speech recognition scores with the extents of HF SNHL and with the $1 \mathrm{kHz}$ gap detection thresholds were studied across all participants. We found that the time-compressed speech recognition scores were significantly affected by and correlated with the extents of HF SNHL. The time-compressed speech recognition scores also correlated with the $1 \mathrm{kHz}$ gap detection thresholds except when the compression ratio of speech was 0.8 under quiet condition. Above all, the extents of HF SNHL were significantly correlated with the $1 \mathrm{kHz}$ gap thresholds.
\end{abstract}

\section{Introduction}

In ENT clinical, patients with high-frequency sensorineural hearing loss (HF SNHL) always complain about the intelligibility of the fast speech. Sensorineural hearing loss (SNHL) is very commonly encountered in the clinic. Researches showed that compared to the normal-hearing $(\mathrm{NH})$ individuals, the ability to comprehend speech in noise decreased in the SNHL individuals [1-3]. Low-intensity signals masked in speech cannot be perceived by those with SNHL, rendering poor speech recognition. Although speech contains a wide range of frequencies [4], according to Ardoint and Lorenzi [5], the most important frequency range in terms of speech perception is $1-2 \mathrm{kHz}$. Usually, the SNHL begins at high frequencies and slowly spreads to lower frequencies. Once SNHL extends into the low-frequency region $(1-2 \mathrm{kHz})$, the speech recognition ability of SNHL individuals becomes even worse.

Of all those with SNHL, even individuals with only HF SNHL usually complain about the intelligibility of fast speech, especially in noise. Accumulated evidence shows that the speech recognition scores of HF SNHL patients with normal low-frequency hearing are poorer than those of $\mathrm{NH}$ individuals, even when speech stimuli are limited to low frequencies [6-10]. Age also played an important role in speech perception [11]. Leigh-Paffenroth and Elangovan [12] found significant poorer temporal processing in the low-frequency regions (with normal thresholds) in middleaged individuals even without HF SNHL, compared to the younger individuals. Fullgrabe et al. [13] found declines in speech perception in older persons compared to the youth persons, even the audiometric sensitivities of both were 
within normal ranges. It is necessary to exclude the influence of age and hearing differences in low-frequency region to study the impact of HF SNHL in speech perception in low frequencies. After auditory sensitivity and age were controlled, research suggested that suprathreshold temporal processing deficits did exist $[6,14]$. Others showed that noise-induced HF SNHL affected low-frequency temporal resolution in guinea pigs, even though the thresholds in the low-frequency region were within normal ranges $[15,16]$. In this point of view, the speech perception difficulties that many SNHL individuals experienced probably consist of not only SNHL of the high-frequency region but also the temporal processing disability in the low-frequency region.

Previous studies found that speech recognition ability varied among individuals with different extents of SNHL [12, 17-20]. Andrade et al. [18] reported that the speech recognition thresholds correlated with the extents of SNHL in individuals with nonflat audiograms. Also, self-assessed scores of hearing disability were associated with the puretone thresholds [17, 19, 20]. Notably, Dobie [19] explored the relationships between pure-tone averages (at $0.5,1$, 2 , and $3 \mathrm{kHz}$ ) and self-assessed hearing disability scores of 1001 patients and found no correlation between selfassessed scores and pure-tone averages in patients whose pure-tone averages were below $25 \mathrm{~dB}$ HL. However, a linear correlation was evident between the self-assessed hearing disability scores and pure-tone averages in patients whose pure-tone averages were above $25 \mathrm{~dB}$ HL [19].

However, whether and how HF SNHL affects lowfrequency speech perception and temporal resolution remains largely unknown. In the present work, we grouped patients by cutoff frequency $(1,2$, and $4 \mathrm{kHz})$ of HF SNHL. Thus, the thresholds at and below each cutoff frequency were within normal ranges, and the thresholds beyond the cutoff frequencies were higher than $25 \mathrm{~dB}$ HL. And gap detection tasks were used to evaluate the temporal resolution of lowfrequency region. Speech recognition scores upon delivery of time-compressed sentences under both quiet and noisy conditions and gap detection thresholds were measured and compared between HF SNHL groups with the same cutoff frequency but various degrees of HF SNHL and age-matched NH group.

\section{Materials and Methods}

2.1. Participants. A total of 76 individuals were recruited, including $65 \mathrm{HF}$ SNHL patients and $11 \mathrm{NH}$ individuals. All HF SNHL participants were recruited from the Department of Otolaryngology Head and Neck Surgery at Shanghai Jiao Tong University Affiliated Sixth People's Hospital, and $\mathrm{NH}$ individuals from the staff of the same hospital. No neurological, psychiatric, or other disorders that would undermine speech recognition ability were identified in all participants including the HF SNHL participants. The program was approved by the Ethics Committee of Shanghai Jiao Tong University Affiliated Sixth People's Hospital. All participants gave written informed consent prior to study commencement.
All participants were native Mandarin-speaking Chinese. All $\mathrm{NH}$ individuals had pure-tone thresholds $25 \mathrm{~dB} \mathrm{HL}$ or less at all octave frequencies between 250 and $8000 \mathrm{~Hz}$, in both ears. HF SNHL patients were rigorously selected according to the following criteria: (1) symmetrical SNHL, with threshold differences of $15 \mathrm{~dB}$ or less (at all frequencies) between both ears for more than 6 months; (2) puretone thresholds of $25 \mathrm{~dB} \mathrm{HL}$ or less, both at and below the cutoff frequencies; (3) pure-tone thresholds $>25 \mathrm{~dB}$ HL above the cutoff frequencies; and (4) type A or Ad type tympanograms.

HF SNHL patients were grouped by the HF SNHL cutoff frequencies evident on audiograms (e.g., 1, 2, and $4 \mathrm{kHz}$ ). In each of these three groups, patients were further subdivided into those with mild (25-40 dB HL), moderate (41-60 dB $\mathrm{HL}$ ), and severe (>60 dB HL) HF SNHL subgroups, defined by the means of averaged pure-tone threshold across frequencies higher than the cutoff frequency. Thus, finally, we formed eight HF SNHL groups, including mild, moderate, and severe groups with cutoff frequencies at 1 and $2 \mathrm{kHz}$ and mild and moderate groups with cutoff frequency at $4 \mathrm{kHz}$, and one $\mathrm{NH}$ group. The means and standard deviations of the auditory thresholds of the tested ears for all groups are shown in Figure 1. Demographic data of all groups are shown in Table 1.

2.2. Stimuli and Procedure. The gap detection task was measured in a three-interval forced-choice procedure. For the gap marker, white noise was low-pass filtered at cutoff frequencies of 1,2 , and $4 \mathrm{kHz}$, respectively, via 3000thorder finite impulse response filter with an approximately $-116 \mathrm{~dB} /$ octave filter slope.

In brief, a three-interval forced-choice program had been run on MATLAB software (version 7.0). Three buttons were presented on a monitor to the participant who was asked to indicate which one of the three stimuli was different (i.e., which of the three stimuli was inserted with a gap). As each of the three stimuli playing, the corresponding button was highlighted in red (from left to right). Participant was instructed to click one of the three buttons with the mouse as a response after each presentation of three signals. The next trial was initiated after an answer was given. All subjects were trained to be familiar with the procedure before formal test. The training would last until their performances reached platforms, respectively. No feedback was given to the subject throughout the test. The gap varying in size from 20 to $1 \mathrm{~ms}$ was embedded in the middle of one of the three noise bursts (total duration: $1000 \mathrm{~ms}$ for each). The gap was shaped using a $1 \mathrm{~ms}$, raised cosine envelope. Each test, commenced with a gap of $20 \mathrm{~ms}$, was followed by a down sequence (in $2 \mathrm{~ms}$ steps) until the first erroneous answer was recorded. The two-down, one-up procedure was then adopted (with a gap step size of $1 \mathrm{~ms}$ ) until the appointed reversals were reached. In gap detection tests, the frequency spectra of the gap markers tested in the HF SNHL groups differed. For example, $4 \mathrm{kHz}$ group members were tested separately with 1,2 , and $4 \mathrm{kHz}$ gap markers. Those of the $2 \mathrm{kHz}$ groups were tested using 1 and $2 \mathrm{kHz}$ gap markers. For those of the $1 \mathrm{kHz}$ groups, only the $1 \mathrm{kHz}$ gap marker test was tested. 


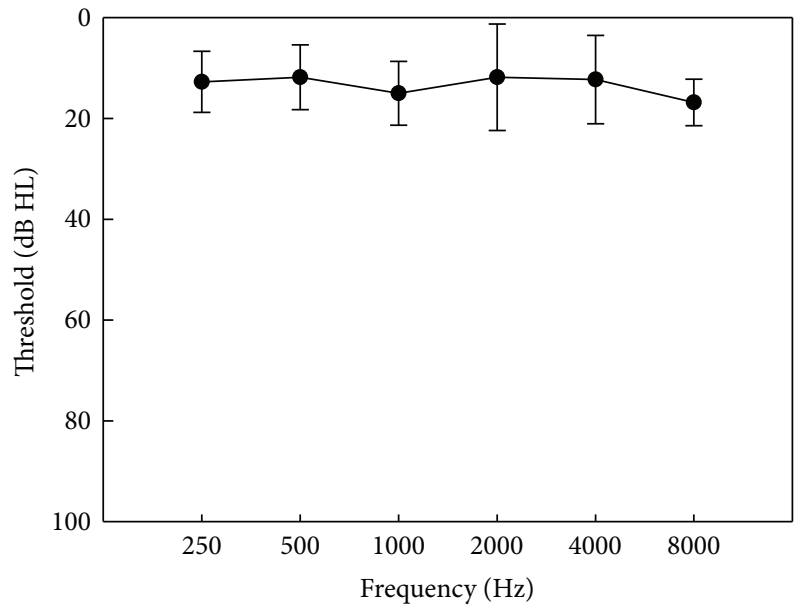

$\mathrm{NH}$ group $n=11$

(a)

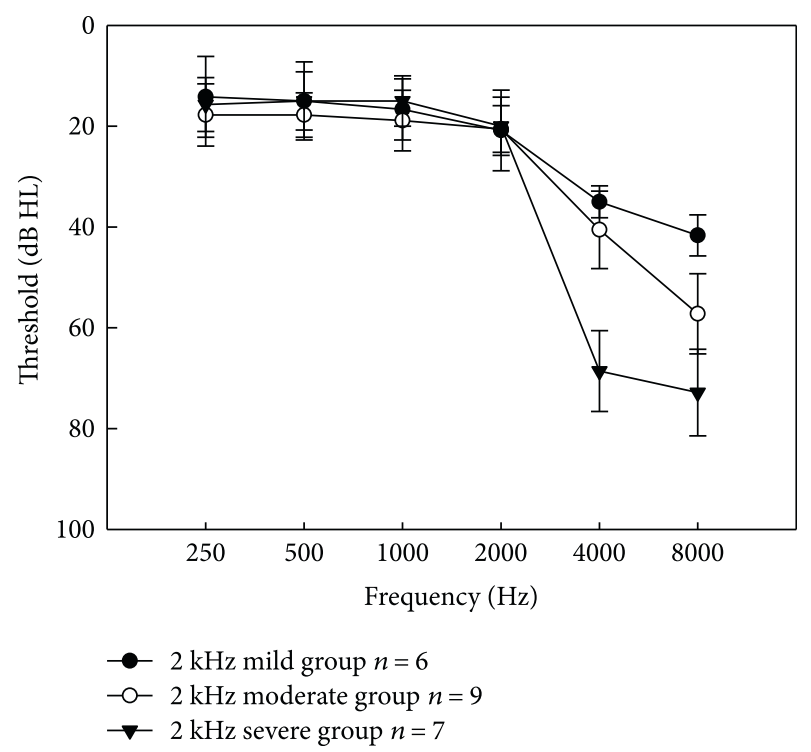

(c)

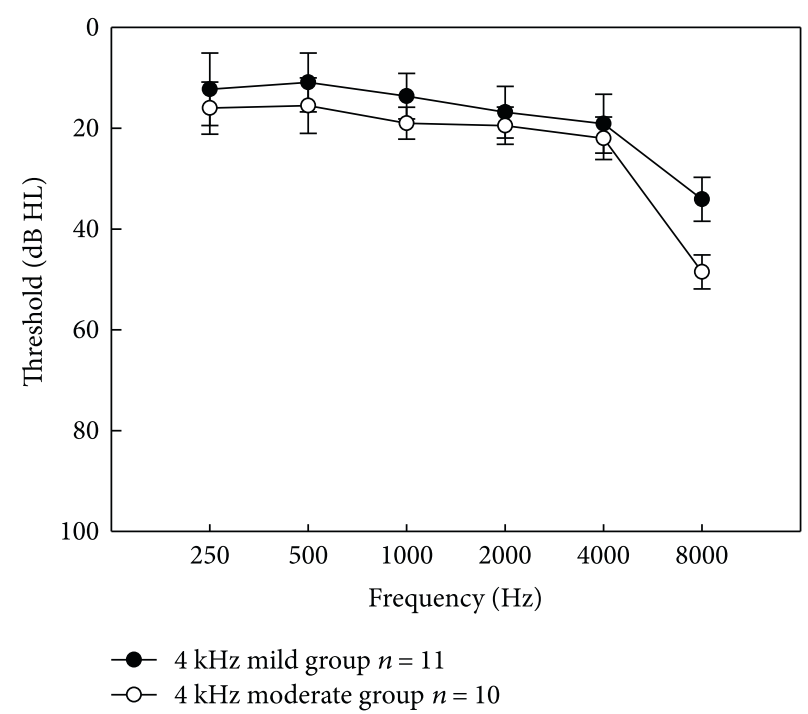

(b)

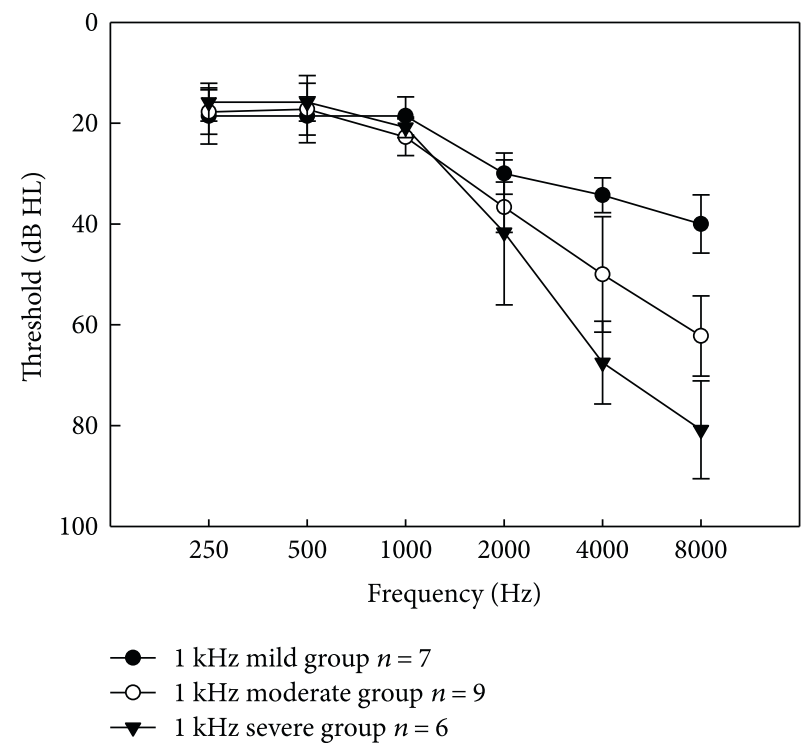

(d)

Figure 1: Mean audiometric thresholds (dB HL), with standard deviations, for each group. The audiometric thresholds of the tested ears for the normal hearing $(\mathrm{NH})$ and 4, 2, and $1 \mathrm{kHz}$ HF SNHL groups are shown in (a), (b), (c) and (d), respectively.

TABle 1: Demographic data for the NH group and HF SNHL subgroups.

\begin{tabular}{lccc}
\hline Group & Male & Female & Age mean \pm SD (yrs) \\
\hline $\mathrm{NH}$ & 2 & 9 & $45.6 \pm 13.5$ \\
$4 \mathrm{kHz}$ mild & 5 & 6 & $51.2 \pm 8.5$ \\
$4 \mathrm{kHz}$ moderate & 5 & 5 & $48.3 \pm 5.3$ \\
$2 \mathrm{kHz}$ mild & 3 & 3 & $52.0 \pm 5.4$ \\
$2 \mathrm{kHz}$ moderate & 3 & 6 & $46.4 \pm 7.9$ \\
$2 \mathrm{kHz}$ severe & 3 & 4 & $52.9 \pm 11.3$ \\
$1 \mathrm{kHz}$ mild & 3 & 4 & $42.6 \pm 8.2$ \\
$1 \mathrm{kHz}$ moderate & 4 & 5 & $52.7 \pm 13.4$ \\
$1 \mathrm{kHz}$ severe & 3 & 3 & $51.0 \pm 8.6$ \\
\hline
\end{tabular}

NH: normal hearing; SD: standard deviation.
Speech perception was assessed using the Mandarin version of the Hearing in Noise Test (MHINT) of the House Ear Institute [21], representing a daily and communicative style of speech, which could be easily understood by native Mandarin-speaking listeners with various degrees of education. Speech was time-compressed using Praat software (version 5.3), without any significant change in the power spectrum [22]. We used three compression ratios: $0.6,0.8$, and 1.0 that of the normal speech rate (the compression ratio of 1.0, namely, was normal speech rate). Speech recognition tests were run under both quiet and noisy [signal-to-noise ratio (SNR): $-5 \mathrm{~dB}$ ] conditions.

All test signals were presented at $75 \mathrm{~dB}$ SPL under both quiet and noisy conditions and were delivered monaurally through Sennheiser HD580 headphones. Only right ears were tested, and a $40 \mathrm{~dB}$ SPL speech-shaped noise was 
conducted to the left ears as masker all along the tests. To create noisy conditions, a speech-shaped noise of the same spectrum as that of the MHINT sentence was presented with SNR at $-5 \mathrm{~dB}$. The noise began $500 \mathrm{~ms}$ before the sentence and continued for $500 \mathrm{~ms}$ after the sentence had concluded. A complete set of tests required approximately $30 \mathrm{~min}$. Practice was conducted before each test, and feedback was provided. After practice, each participant achieved stable recognition scores. During a speech recognition test, each sentence was played only once, and no feedback was given. The same methods were also applied by Feng et al. [14].

\section{Results}

3.1. Age Matching and Pure-Tone Thresholds of the NH and HF SNHL Groups. One-way analysis of variance (ANOVA) showed that the mean ages of all nine groups did not differ significantly $\left(F_{(8,75)}=1.097, p=0.376\right)$.

Comparisons of the averaged thresholds across the frequencies with normal thresholds in all groups showed that the thresholds of frequencies exhibiting normal hearing did not differ significantly among the groups $\left(F_{(8,75)}=1.899, p=0.075\right)$.

3.2. Gap Detection Task. The gap thresholds of groups varying in terms of gap marker cutoff frequency are shown in Figure 2. The gap thresholds of the gap markers with different cutoff frequencies for the same listener group were compared firstly. Paired $t$-tests showed that the gap thresholds of $1 \mathrm{kHz}$ gap marker were significantly higher than those of $2 \mathrm{kHz}$ gap marker for $2 \mathrm{kHz}$ mild HF SNHL group $(t=5.349, p=0.003), 2 \mathrm{kHz}$ moderate HF SNHL group $(t=10.639, p<0.001)$, and $2 \mathrm{kHz}$ severe HF SNHL group $(t=7.22, p<0.001)$. One-way repeated ANOVA showed significant main effects of cutoff frequencies of gap marker on gap thresholds of $4 \mathrm{kHz}$ mild HF SNHL group $\left(F_{(2,20)}=19.334, p<0.001\right), 4 \mathrm{kHz}$ moderate HF SNHL group $\left(F_{(2,18)}=21.063, p<0.001\right)$, and NH group $\left(F_{(2,20)}=57.133, p<0.001\right)$; the post hoc analyses (LSD tests) revealed that gap thresholds of $1 \mathrm{kHz}$ gap marker, $2 \mathrm{kHz}$ gap marker, and $4 \mathrm{kHz}$ gap marker differed from each other significantly for the three groups, respectively. Generally, the gap thresholds of all groups gradually decrease as cutoff frequencies of the gap marker increase gradually.

Then, data derived from different groups with the same gap marker frequency were analyzed by one-way ANOVA. There was a significant difference when the cutoff frequency of gap marker is $1 \mathrm{kHz}\left(F_{(8,75)}=2.189, p=0.039\right)$; the post hoc analysis (LSD test) revealed that the gap thresholds of the $\mathrm{NH}$ group and $4 \mathrm{kHz}$ mild HF SNHL group were significantly lower than those of the $1 \mathrm{kHz}$ mild HF SNHL group, $1 \mathrm{kHz}$ moderate HF SNHL group, and $1 \mathrm{kHz}$ severe HF SNHL group. And there was also a significant difference when the cutoff frequency of gap marker is $4 \mathrm{kHz}$ $\left(F_{(2,31)}=3.515, p=0.043\right)$; the post hoc analysis (LSD test) revealed that the gap thresholds of the $\mathrm{NH}$ group were significantly lower than those of the $4 \mathrm{kHz}$ moderate HF SNHL group with $4 \mathrm{kHz}$ gap marker. However, no difference was evident with $2 \mathrm{kHz}$ gap marker $\left(F_{(5,53)}=0.231, p=0.947\right)$.
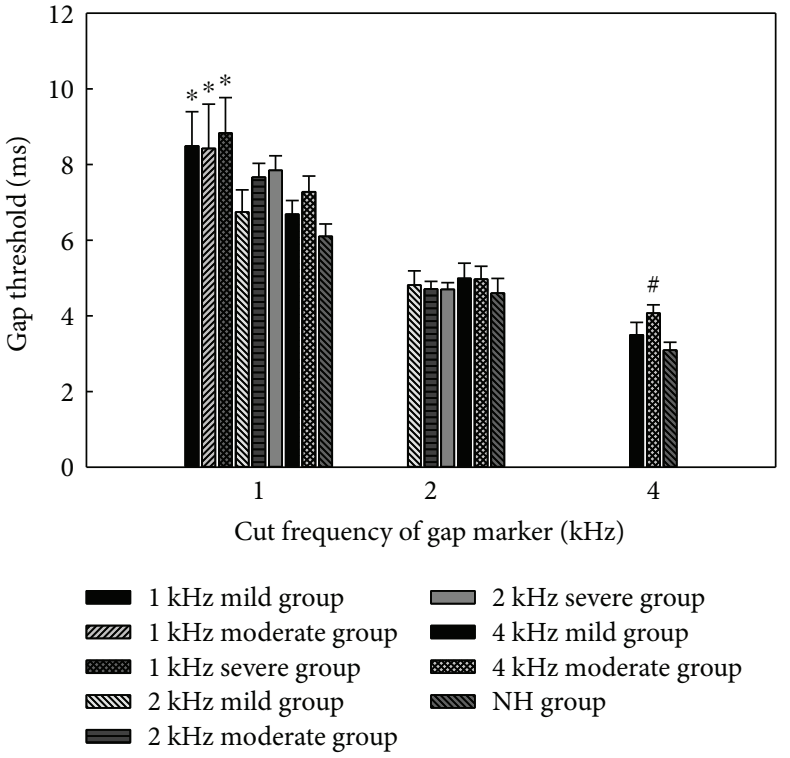

Figure 2: The mean gap thresholds in the high-frequency sensorineural hearing loss (HF SNHL) groups and normal-hearing $(\mathrm{NH})$ group. The error bars indicate standard errors. * indicates gap thresholds of the $1 \mathrm{kHz}$ mild, moderate, and severe HF SNHL groups with $1 \mathrm{kHz}$ gap marker which were significantly higher than those of the $\mathrm{NH}$ group and the $4 \mathrm{kHz}$ mild HF SNHL group; \# indicates gap thresholds of the $4 \mathrm{kHz}$ moderate HF SNHL group which were significantly higher than those of the $\mathrm{NH}$ group for $4 \mathrm{kHz}$ gap marker.

When presented with gap marker of the same cutoff frequency, in general, the gap thresholds of various groups tended to be higher if the range of HF SNHL was wider or the degrees of hearing impairment were higher.

3.3. Time-Compressed Speech Recognition. The original scores under quiet and noisy conditions are shown in Figures 3 and 4, respectively. Overall, the speech recognition scores of all groups decreased as the time compression ratio fell from 1.0 (normal speech rate) to 0.6 and the scores were lower under noisy conditions than those under quiet conditions at the same time compression ratio.

Before analysis, all speech recognition scores were arcsine-transformed to avoid ceiling or floor effects. Data from the $\mathrm{NH}$ and eight HF SNHL groups in quiet were subjected to two-way repeated-measures ANOVA to test the effects of group and time compression ratio on speech recognition. The effects of group and compression ratio were both significant: $F_{\text {group(8,67) }}=5.368, p<0.001$ and $F_{\text {compression(2,134) }}=114.028, p<0.001$. There was a statistically significant two-way interaction between group and time compression ratio $\left(F_{(16,134)}=2.130, p=0.010\right)$. The LSD method was applied in post hoc comparisons, to explore the effect of the extent of HF SNHL on speech recognition scores in quiet. When speech compression ratio was 0.6, all HF SNHL groups scored significantly lower than the $\mathrm{NH}$ group except the $2 \mathrm{kHz}$ mild and severe HF SNHL groups and $4 \mathrm{kHz}$ mild HF SNHL group; when speech compression ratio was 0.8 , the $\mathrm{NH}$ group scored significantly higher than the $1 \mathrm{kHz}$ moderate and severe HF SNHL groups and $4 \mathrm{kHz}$ 


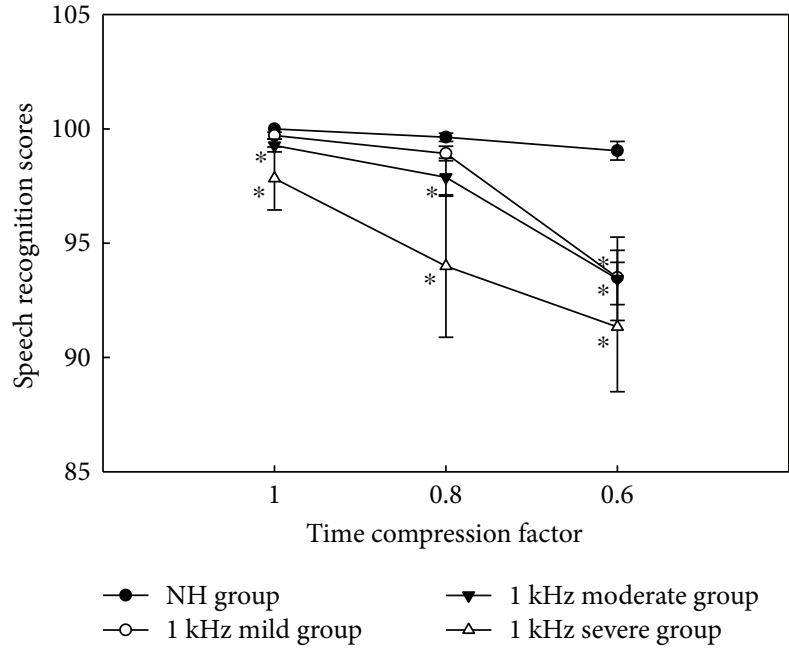

(a)

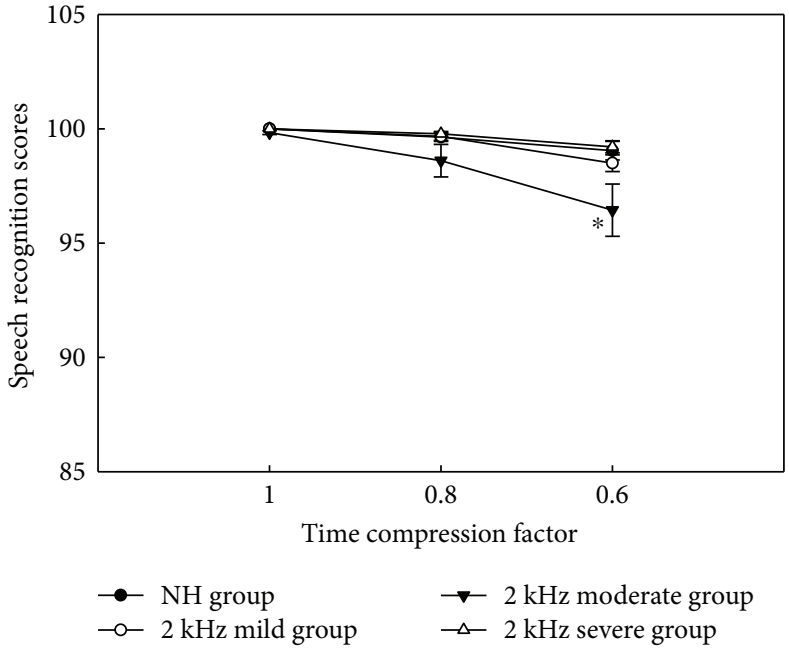

(b)

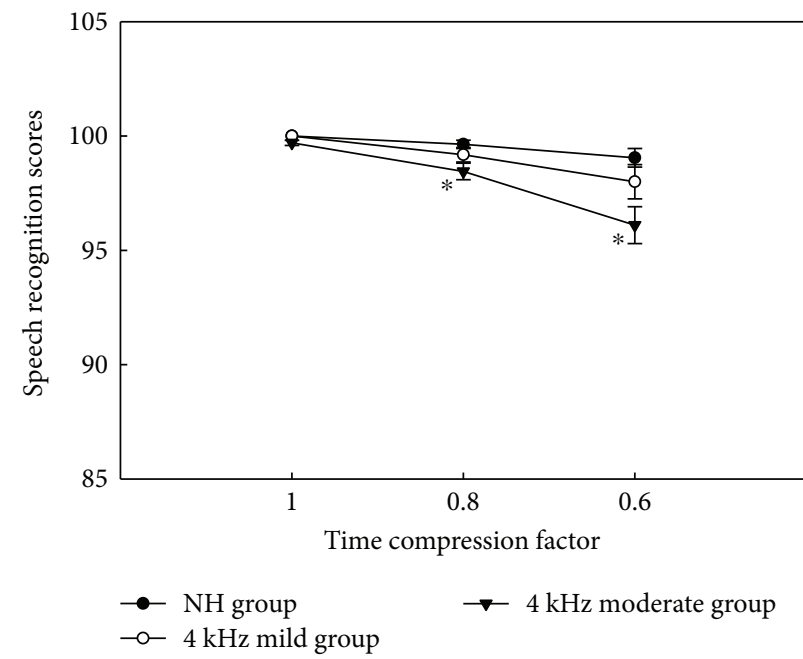

(c)

FIGURE 3: Speech recognition scores under quiet conditions for the normal-hearing (NH) and high-frequency sensorineural hearing loss (HF SNHL) groups, as a function of the time compression ratio. The scores for the 1, 2, and $4 \mathrm{kHz}$ HF SNHL groups are shown in (a), (b), and (c), respectively. The error bars indicate standard errors. * indicates significant difference of speech recognition scores when compared with that of the $\mathrm{NH}$ group at the same time compression factor.

moderate HF SNHL group, while the $\mathrm{NH}$ group scored significantly higher than the $1 \mathrm{kHz}$ moderate and severe $\mathrm{HF}$ SNHL groups when speech compression ratio was 1.0. The differences among the $1 \mathrm{kHz}$ mild, moderate, and severe HF SNHL groups were not statistically significant when speech compression ratio was 0.6 or 1.0 , but the scores of the $1 \mathrm{kHz}$ mild $\mathrm{HF}$ SNHL groups were significantly higher than the $1 \mathrm{kHz}$ severe HF SNHL groups when speech compression ratio was 0.8 ; the differences among the $2 \mathrm{kHz}$ mild, moderate, and severe HF SNHL groups and the differences between the $4 \mathrm{kHz}$ mild and moderate HF SNHL groups were not statistically significant for all three speech compression ratios.

A two-way repeated-measures ANOVA was used to evaluate the effects of group and time compression ratio on speech recognition in noise. The effects of group and compression ratio were both significant: $F_{\text {group }(8,67)}=11.541$, $p<0.001$ and $F_{\text {compression(2,134) }}=144.785, p<0.001$. There was a statistically significant two-way interaction between group and time compression ratio $\left(F_{(16,134)}=4.434, p \leq\right.$ $0.001)$. The LSD method was used in post hoc comparisons to explore the effect of the extent of HF SNHL on speech recognition scores in noise. When speech compression ratio was 0.8 and 1.0, the differences between scores of the $\mathrm{NH}$ group and all HF SNHL groups were significant; when speech compression ratio was 0.6 , the $\mathrm{NH}$ group scored significantly higher than all HF SNHL groups except the $2 \mathrm{kHz}$ mild HF SNHL group. The differences among the $1 \mathrm{kHz}$ mild, moderate, and severe HF SNHL groups were not statistically significant when speech compression ratio was 0.6 or 0.8 , but the scores of the $1 \mathrm{kHz}$ mild HF SNHL groups were significantly higher than those of $1 \mathrm{kHz}$ severe HF SNHL groups when speech compression ratio was 1.0; the $2 \mathrm{kHz}$ mild HF SNHL group scored significantly higher 


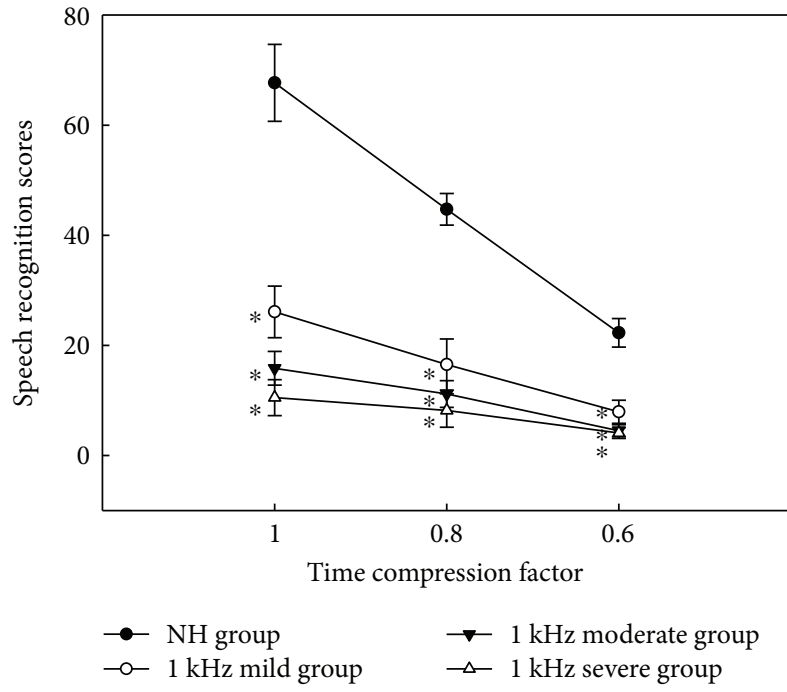

(a)

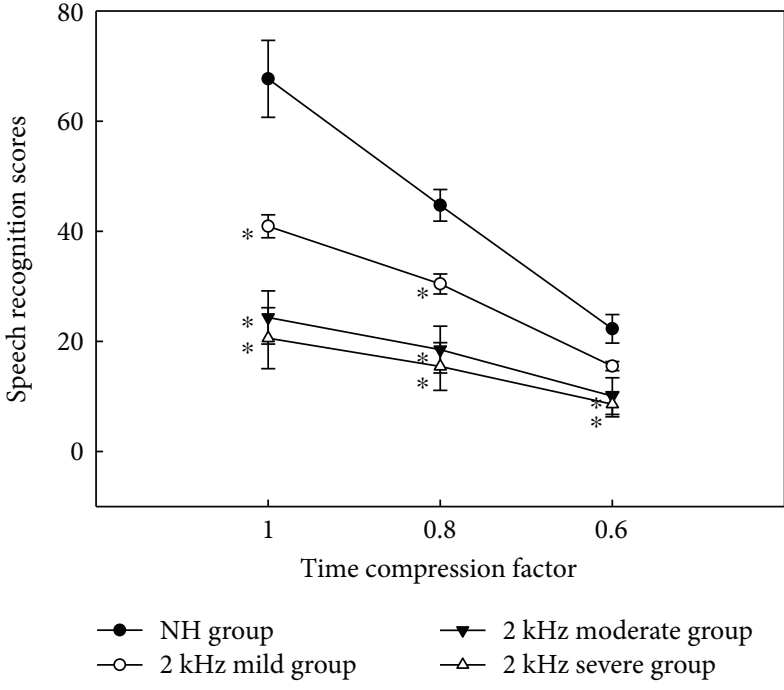

(b)

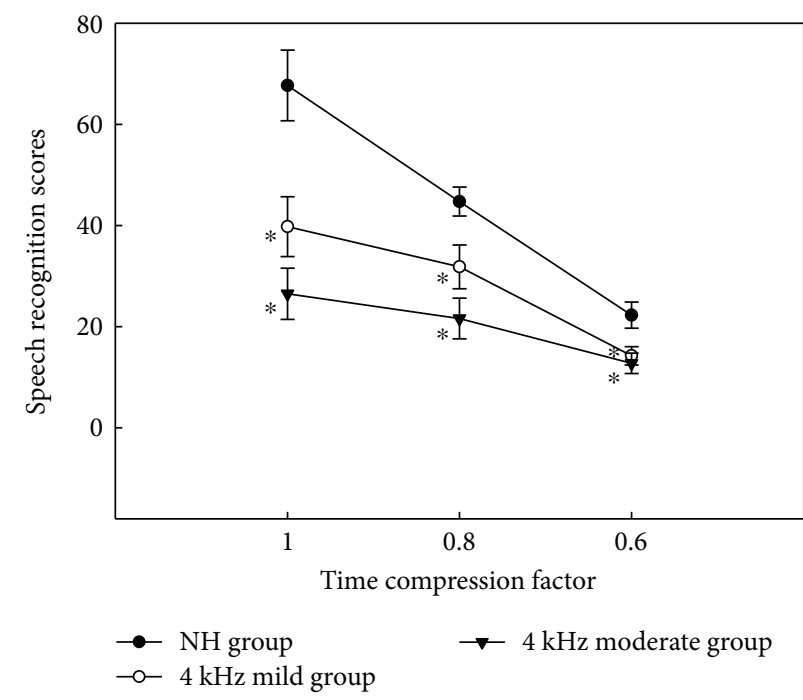

(c)

FIGURE 4: Speech recognition scores under noisy conditions (SNR $=-5 \mathrm{~dB})$ for the normal-hearing $(\mathrm{NH})$ and high-frequency sensorineural hearing loss (HF SNHL) groups, as a function of the time compression ratio. The scores for the 1, 2, and $4 \mathrm{kHz}$ HF SNHL groups are shown in (a), (b), and (c), respectively. The error bars indicate standard errors. * indicates significant difference of speech recognition scores when compared with that of the $\mathrm{NH}$ group at the same time compression factor.

than the $2 \mathrm{kHz}$ moderate and severe HF SNHL groups when speech compression ratio was 0.6 and 0.8 , and the $2 \mathrm{kHz}$ mild HF SNHL group scored significantly higher than the $2 \mathrm{kHz}$ severe HF SNHL group when speech compression ratio was 1.0. The differences between the $4 \mathrm{kHz}$ mild and moderate HF SNHL groups were not statistically significant for all three speech compression ratios.

As a whole, at the same time compression ratio, the scores of the NH group were better than those of any HF SNHL group, and the scores of those with HF SNHL decreased as the degree of HF SNHL increased, which was more obvious under noisy conditions.

3.4. Correlation Analysis. We explored relationships between time-compressed speech recognition scores, pure-tone HF averages, and gap detection thresholds, by calculating Pearson correlations as follows: (1) between pure-tone HF averages (for NH groups, the average was calculated across 2,4 , and $8 \mathrm{kHz}$; for HF SNHL groups, the average was calculated across frequencies where exhibited hearing loss) and timecompressed speech recognition scores; (2) between puretone $\mathrm{HF}$ averages and the $1 \mathrm{kHz}$ gap detection thresholds; and, (3) between the time-compressed speech recognition scores and the $1 \mathrm{kHz}$ gap detection thresholds (all participants underwent $1 \mathrm{kHz}$ gap detection testing). As shown in Table 2, the pure-tone HF averages were significantly correlated with time-compressed speech recognition scores at compression ratios of both 0.6 and 0.8 , and normal speech recognition scores (all $p$ values $<0.05$ ), under both quiet and noisy conditions. What is noteworthy is that pure-tone 
TABLE 2: Correlation analysis between pure-tone averages of high-frequency hearing loss and speech recognition scores with different compression and different test backgrounds.

\begin{tabular}{|c|c|c|c|c|c|c|}
\hline & \multicolumn{3}{|c|}{ Compression ratio of speech in quiet } & \multicolumn{3}{|c|}{ Compression ratio of speech in noise } \\
\hline & 1 & 0.8 & 0.6 & 1 & 0.8 & 0.6 \\
\hline Pearson correlation & -0.279 & -0.269 & -0.329 & -0.694 & -0.627 & -0.536 \\
\hline$p$ value & $0.015^{*}$ & $0.019^{*}$ & $0.004^{*}$ & $<0.001^{*}$ & $<0.001^{*}$ & $<0.001^{*}$ \\
\hline
\end{tabular}

For NH groups, the average was calculated across 2, 4, and $8 \mathrm{kHz}$; for HF SNHL groups, the average was calculated across frequencies where exhibited hearing loss. * indicates $p$ values smaller than 0.05 .

TABLE 3: Correlation analysis between $1 \mathrm{kHz}$ gap thresholds and speech recognition scores with different compression ratios under different test backgrounds.

\begin{tabular}{lccccc}
\hline & \multicolumn{3}{c}{ Compression ratio of speech in quiet } & \multicolumn{3}{c}{ Compression ratio of speech in noise } \\
& 1 & 0.8 & 0.6 & 1 & 0.8 \\
\hline Pearson correlation & -0.231 & -0.185 & -0.427 & -0.388 & -0.394 \\
$p$ value & $0.045^{*}$ & 0.109 & $<0.001^{*}$ & $0.001^{*}$ & -0.381 \\
\hline
\end{tabular}

* indicates $p$ values smaller than 0.05 .

HF averages were significantly correlated with the $1 \mathrm{kHz}$ gap detection thresholds (Pearson correlation $=0.367, p=0.001$ ). In Table 3, the results showed that $1 \mathrm{kHz}$ gap thresholds were significantly correlated with the speech recognition scores at all compression ratios under noisy conditions (all $p$ values $\leq 0.001$ ). This was also true under quiet conditions for both normal speech and that at a 0.6 compression ratio $\left(p_{0.6 \text { compression }} \leq 0.001, p_{\text {normal speed }}=0.045\right)$.

\section{Discussion}

Our primary purpose in the present study was to explore whether and how HF SNHL affected time-compressed speech perception and gap detection in low-frequency region with normal auditory threshold. We found that the timecompressed speech recognition scores of the HF SNHL group were poorer than those of the $\mathrm{NH}$ group and decreased as the extent of HF SNHL increased in patients with the same cutoff frequency. Generally, the recognition scores of patients with severe HF SNHL were poorer than the scores of those with moderate HF SNHL, which in turn were poorer than those of patients with mild HF SNHL at the same cutoff frequencies, under both quiet and noisy conditions (Figures 3 and 4).

As shown in Table 2, pure-tone averages of HF SNHL were significantly correlated with the time-compressed speech recognition scores. These results suggested that the ability to recognize time-compressed speech was affected by HF SNHL and correlated with the extent of HF SNHL. These results are similar to those of our previous study and indeed extend our earlier work [14]. We previously showed that the time-compressed speech recognition scores of the HF SNHL subjects were poorer than those of $\mathrm{NH}$ individuals [14]. However, the effect of the extent of HF SNHL on speech recognition was not explored in detail. Therefore, in the present study, we focused on the effect of varying levels of HF SNHL on time-compressed speech recognition abilities.

Indeed, the extent of HF SNHL affects the ability of speech recognition. Moore [23] found that, in individuals with cochlear hearing loss of up to approximately $45 \mathrm{~dB}$, a change in audibility was the single most important contributor to speech perception problems. However, when the extent of hearing loss was greater, poor discrimination of suprathreshold stimuli also became of major importance. Nimitbunnasarn et al. [24] examined tonal identification in Thai speakers with normal hearing and different extents of SNHL. Identification ability was affected by SNHL per se and the extent thereof [24]. Jerger et al. [25] explored correlations among speech recognition performance, the pure-tone hearing level, and the age of individuals with SNHL. The relationship between the extent of hearing loss and speech recognition score was strongest in older individuals with SNHL. Other studies sought correlations between self-assessed hearing inventories and individual pure-tone thresholds [12,17, 19] or between speech reception and pure-tone thresholds [20]. All data suggested that the extents of SNHL correlated with the speech recognition scores. Our results are consistent with such findings and suggest that the extents of HF SNHL are also correlated with the time-compressed speech recognition scores. Similar hearing configurations that vary in the extent of hearing loss may impact speech recognition differently, especially when speech is fast. More severe speech disruption is evident as the severity of hearing loss rises.

It is worth noting that the pure-tone averages of HF SNHL correlated with the $1 \mathrm{kHz}$ gap thresholds significantly, which indicates that the HF SNHL could impair the temporal resolution of the low-frequency region. This is in line with our previous research results, which suggests that HF SNHL exerted an off-channel effect on temporal processing ability in the low-frequency region of the auditory system, whether in guinea pigs or humans $[14,15]$. This off-channel effect may contribute to the difficulty, which is experienced by patients with normal hearing in low frequencies but suffered from HF SNHL, of perceiving the time-compressed speech [14] and temporal fine structure speech [6].

The relationship between speech recognition and temporal resolution, another focus of the present study, remains unclear. Previous studies suggested that temporal resolution 
played an important role in speech recognition [26-29]. However, no influence of any temporal processing ability, such as gap detection, on speech perception, has been conclusively shown [11].

To determine whether the gap detection threshold correlated with time-compressed speech recognition ability, we carefully controlled for age and the extent of HF SNHL. As shown in Table 3, the gap thresholds with $1 \mathrm{kHz}-\mathrm{low}$ pass-filtered noise were negatively correlated with the time-compressed speech recognition scores, suggesting that speech recognition ability was also affected by temporal resolution of the low-frequency region, even whose auditory sensitivity was normal.

\section{Conclusions}

Even when age was controlled, the extent of HF SNHL impacted the ability to recognize compressed speech. The greater the extent of HF SNHL was, the poorer the speech recognition ability was. The significant correlation between the extents of HF SNHL and gap detection thresholds implied that there was probable off-channel mechanism underlying. The decrease of time-compressed speech recognition ability may be partly attributable to the increased thresholds in gap detection task, which signified the deterioration of suprathresholdly temporal resolution.

\section{Conflicts of Interest}

The authors declare that there is no competing interest relevant to the publication of this paper.

\section{Authors' Contributions}

Bei Li and Yang Guo contributed equally to this paper.

\section{Acknowledgments}

The study was supported by a grant from the National Natural Science Foundation of China (81771015), Shanghai Municipal Education Commission-Gaofeng Clinical Medicine Grant Support (20152526), and Three-Year Action Program on Promotion of Clinical Skills and Clinical Innovation for Municipal Hospitals (16CR4027A) to Yanmei Feng and a grant from the National Natural Science Foundation of China (81530029) to Shankai Yin.

\section{References}

[1] J. R. Dubno, D. D. Dirks, and D. E. Morgan, "Effects of age and mild hearing loss on speech recognition in noise," The Journal of the Acoustical Society of America, vol. 76, no. 1, pp. 87-96, 1984.

[2] A. R. Needleman and C. C. Crandell, "Speech recognition in noise by hearing-impaired and noise-masked normal-hearing listeners," Journal of the American Academy of Audiology, vol. 6, no. 6, pp. 414-424, 1995.

[3] A. J. Klein, J. H. Mills, and W. Y. Adkins, "Upward spread of masking, hearing loss, and speech recognition in young and elderly listeners," The Journal of the Acoustical Society of America, vol. 87, no. 3, pp. 1266-1271, 1990.

[4] B. C. J. Moore, "The role of temporal fine structure processing in pitch perception, masking, and speech perception for normal-hearing and hearing-impaired people," Journal of the Association for Research in Otolaryngology, vol. 9, no. 4, pp. 399-406, 2008.

[5] M. Ardoint and C. Lorenzi, "Effects of lowpass and highpass filtering on the intelligibility of speech based on temporal fine structure or envelope cues," Hearing Research, vol. 260, no. 1-2, pp. 89-95, 2010.

[6] B. Li, L. Hou, L. Xu et al., "Effects of steep high-frequency hearing loss on speech recognition using temporal fine structure in low-frequency region," Hearing Research, vol. 326, pp. 66-74, 2015.

[7] A. C. Leger, D. T. Ives, and C. Lorenzi, “Abnormal intelligibility of speech in competing speech and in noise in a frequency region where audiometric thresholds are near-normal for hearing-impaired listeners," Hearing Research, vol. 316, pp. 102-109, 2014.

[8] A. C. Léger, B. C. J. Moore, and C. Lorenzi, “Abnormal speech processing in frequency regions where absolute thresholds are normal for listeners with high-frequency hearing loss," Hearing Research, vol. 294, no. 1-2, pp. 95-103, 2012.

[9] C. Lorenzi, L. Debruille, S. Garnier, P. Fleuriot, and B. C. J. Moore, "Abnormal processing of temporal fine structure in speech for frequencies where absolute thresholds are normal," The Journal of the Acoustical Society of America, vol. 125, no. 1, pp. 27-30, 2009.

[10] V. Summers, M. J. Makashay, S. M. Theodoroff, and M. R. Leek, "Suprathreshold auditory processing and speech perception in noise: hearing-impaired and normal-hearing listeners," Journal of the American Academy of Audiology, vol. 24, no. 4, pp. 274-292, 2013.

[11] A. Strouse, D. H. Ashmead, R. N. Ohde, and D. W. Grantham, "Temporal processing in the aging auditory system," The Journal of the Acoustical Society of America, vol. 104, no. 4, pp. 2385-2399, 1998.

[12] E. D. Leigh-Paffenroth and S. Elangovan, “Temporal processing in low-frequency channels: effects of age and hearing loss in middle-aged listeners," Journal of the American Academy of Audiology, vol. 22, no. 7, pp. 393-404, 2011.

[13] C. Fullgrabe, B. C. Moore, and M. A. Stone, "Age-group differences in speech identification despite matched audiometrically normal hearing: contributions from auditory temporal processing and cognition," Frontiers in Aging Neuroscience, vol. 6, p. 347, 2014.

[14] Y. Feng, S. Yin, M. Kiefte, and J. Wang, "Temporal resolution in regions of normal hearing and speech perception in noise for adults with sloping high-frequency hearing loss," Ear and Hearing, vol. 31, no. 1, pp. 115-125, 2010.

[15] Y. Feng, S. Yin, and J. Wang, "Deterioration of cortical responses to amplitude modulations of low-frequency carriers after high-frequency cochlear lesion in guinea pigs," International Journal of Audiology, vol. 49, no. 3, pp. 228-237, 2010.

[16] S. K. Yin, Y. M. Feng, Z. N. Chen, and J. Wang, "The effect of noise-induced sloping high-frequency hearing loss on the gap-response in the inferior colliculus and auditory cortex of guinea pigs," Hearing Research, vol. 239, no. 1-2, pp. 126-140, 2008. 
[17] C. H. Chien, T. Y. Tu, A. S. Shiao et al., "Prediction of the pure-tone average from the speech reception and auditory brainstem response thresholds in a geriatric population," ORL, vol. 70, no. 6, pp. 366-372, 2008.

[18] K. C. Andrade, P. L. Menezes, A. T. Carnauba, R. G. Rodrigues, M. C. Leal, and L. D. Pereira, "Non-flat audiograms in sensorineural hearing loss and speech perception," Clinics, vol. 68, no. 6, pp. 815-819, 2013.

[19] R. A. Dobie, "The AMA method of estimation of hearing disability: a validation study," Ear and Hearing, vol. 32, no. 6, pp. 732-740, 2011.

[20] S. Coren and A. R. Hakstian, "The development and crossvalidation of a self-report inventory to assess pure-tone threshold hearing sensitivity," Journal of Speech and Hearing Research, vol. 35, no. 4, pp. 921-928, 1992.

[21] L. L. Wong, S. D. Soli, S. Liu, N. Han, and M. W. Huang, "Development of the Mandarin hearing in noise test (MHINT)," Ear and Hearing, vol. 28, 2 Supplement, pp. 70S-74S, 2007.

[22] P. Boersma and D. Weenink, "Praat: Doing phoenetics by computer [computer program]," in Version 4.3.14 Amsterdam, Institute of Phonetic Sciences, University of Amsterdam; 2005, Netherlands, 2008.

[23] B. C. Moore, "Perceptual consequences of cochlear hearing loss and their implications for the design of hearing aids," Ear and Hearing, vol. 17, no. 2, pp. 133-161, 1996.

[24] C. Nimitbunnasarn, P. Amatyakul, J. Gandour, A. Carney, C. Nimitbunnasarn, and P. Amatyakul, "Tonal confusions in Thai patients with sensorineural hearing loss," Journal of Speech and Hearing Research, vol. 27, no. 1, pp. 89-97, 1984.

[25] J. Jerger, S. Jerger, and F. Pirozzolo, "Correlational analysis of speech audiometric scores, hearing loss, age, and cognitive abilities in the elderly," Ear and Hearing, vol. 12, no. 2, pp. 103-109, 1991.

[26] R. Drullman, J. M. Festen, and R. Plomp, "Effect of temporal envelope smearing on speech reception," The Journal of the Acoustical Society of America, vol. 95, no. 2, pp. 1053-1064, 1994.

[27] K. W. Grant and T. C. Walden, "Understanding excessive SNR loss in hearing-impaired listeners," Journal of the American Academy of Audiology, vol. 24, no. 4, pp. 258-273, 2013.

[28] B. R. Glasberg, B. C. Moore, and S. P. Bacon, "Gap detection and masking in hearing-impaired and normal-hearing subjects," The Journal of the Acoustical Society of America, vol. 81, no. 5, pp. 1546-1556, 1987.

[29] R. S. Tyler, Q. Summerfield, E. J. Wood, and M. A. Fernandes, "Psychoacoustic and phonetic temporal processing in normal and hearing-impaired listeners," The Journal of the Acoustical Society of America, vol. 72, no. 3, pp. 740-752, 1982. 

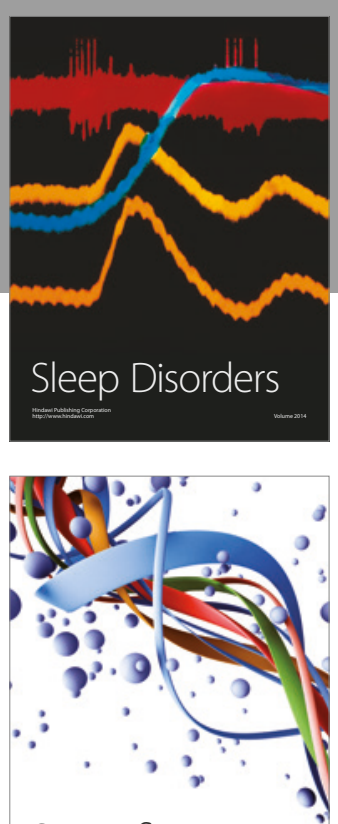

Scientifica
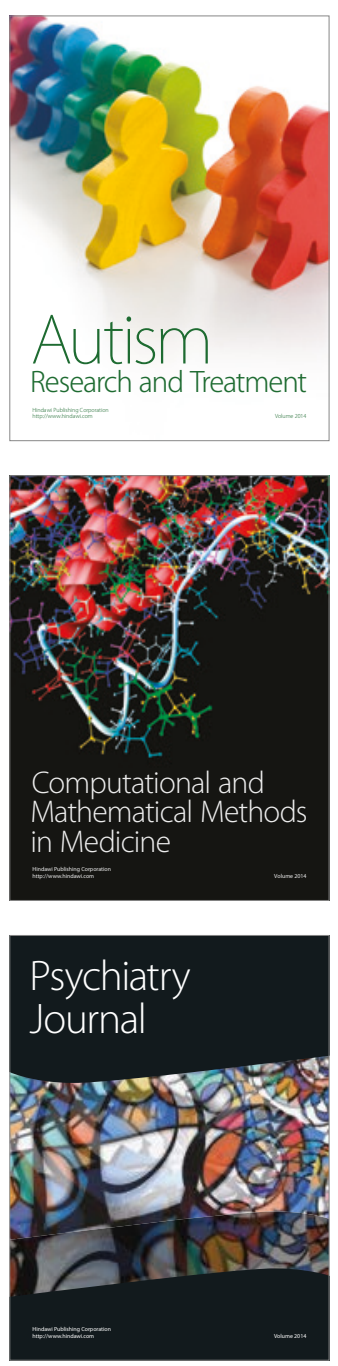
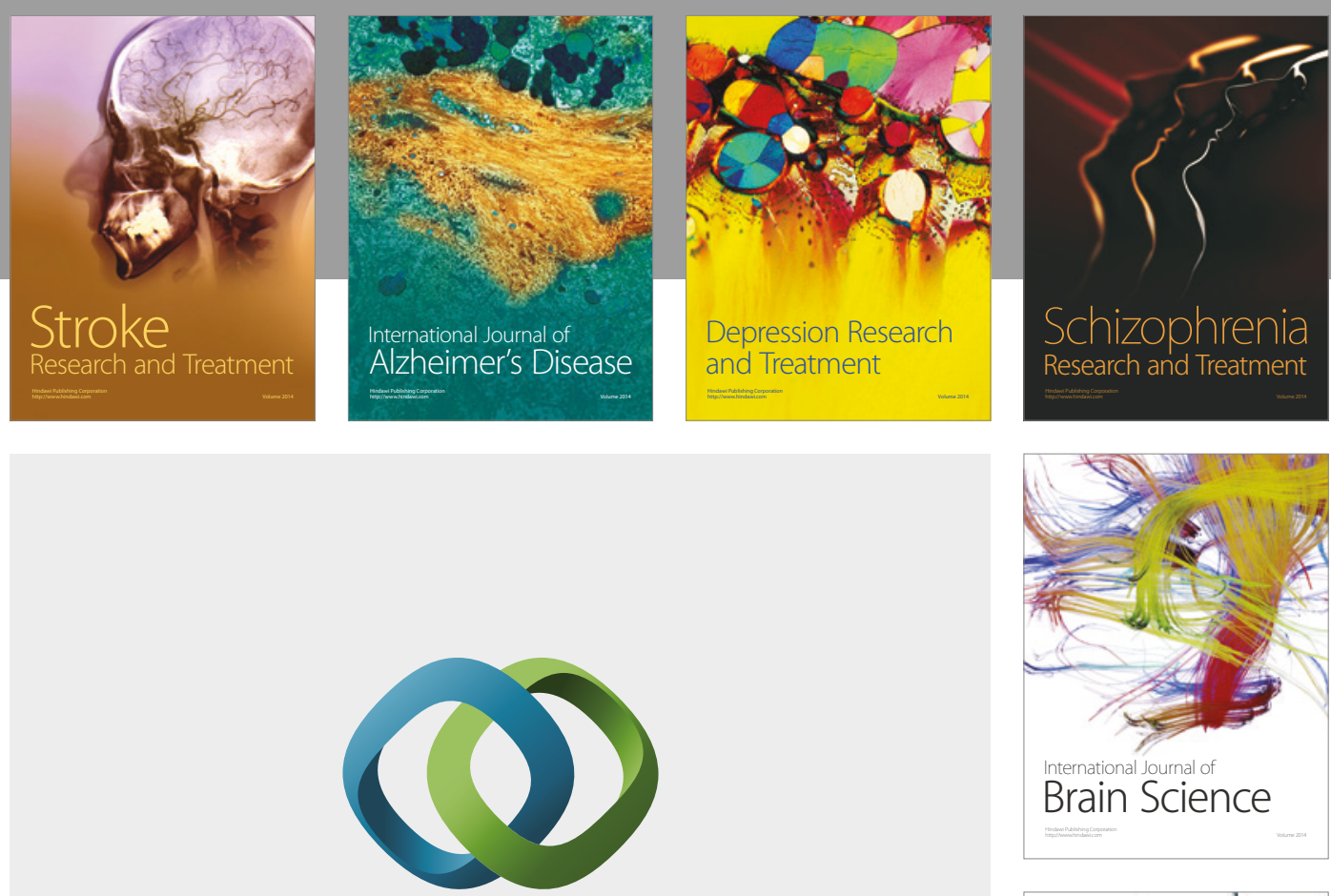

\section{Hindawi}

Submit your manuscripts at

https://www.hindawi.com
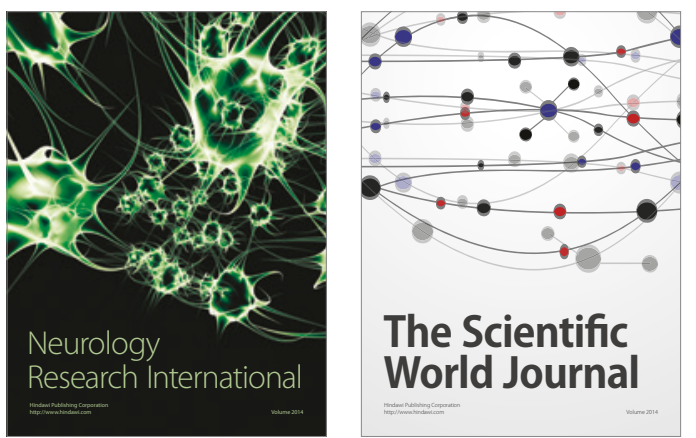

The Scientific World Journal

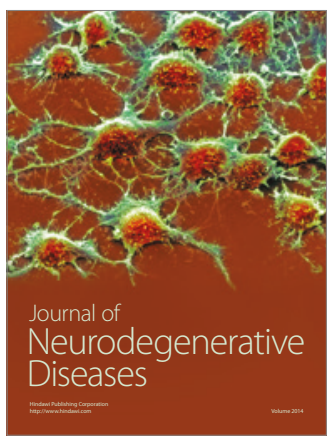

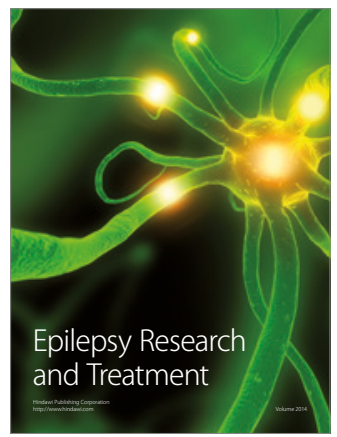

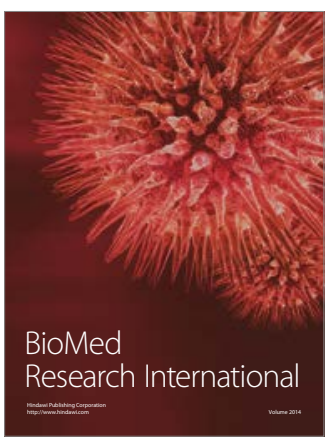

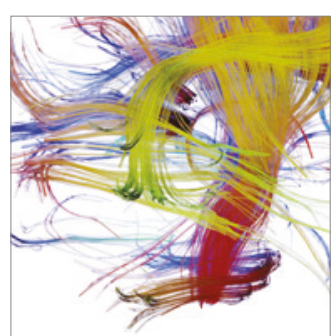

Brain Science

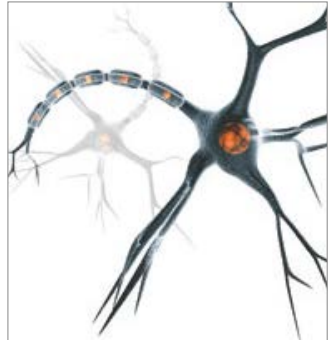

Neural Plasticity
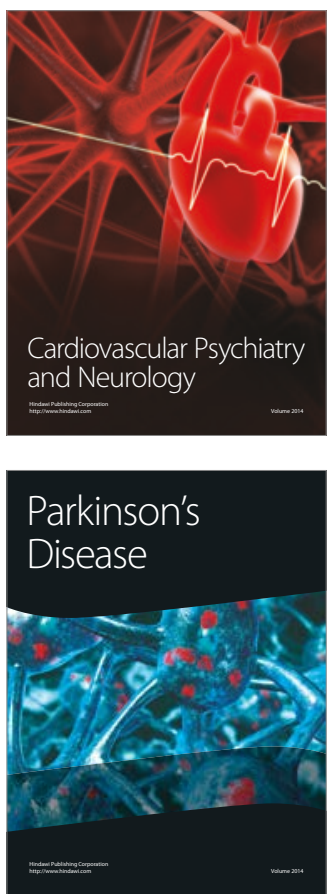